\title{
A MAC Protocol for Wireless Sensors in a Fixed Chain Topology
}

\author{
Jalawi Alshudukhi, Shumao Ou, Peter Ball \\ Department of Computing and Communication Technologies, Oxford Brookes University, Oxford OX33 1HX, U.K. \\ $\{12045494$, sou,pball $\} @$ brookes.ac.uk
}

\begin{abstract}
This paper presents a time division multiple access MAC protocol that is specifically designed for applications requiring periodic sensing of the sensor field. Numerical analysis is conducted to investigate the optimum transmission scheduling based on the signal to interferencenoise-ratio (SINR) for ground level propagation model applied on wireless chain topology. The optimised transmission schedule considers the SINR value to enable simultaneous transmission from multiple nodes. The most significant advantages of this approach are reduced delay and improve the Packet Received Ratio PRR. Simulation is performed to evaluate the proposed protocol for intelligent transport system applications. The simulation results validate the MAC protocol for a fixed chain topology compared with the similar protocols.
\end{abstract}

Keywords-MAC Protocol, Schedule based, Low-power Wireless Sensors, Chain Topology

\section{INTRODUCTION}

Several medium access control (MAC) protocols have been proposed for wireless sensor networks which aim to provide an efficient way of sharing the transmission medium.. The primary constraint of sensor nodes in WSNs is their low battery capacity. Furthermore, sensor nodes are often left unattended after deployment, and this makes the replacement or recharging of their batteries difficult; MAC protocols running on WSNs must consume energy efficiently to achieve a long network lifetime [1].

The sources of energy wastage have been addressed in several studies. These sources include idle listening, collision overhearing and protocol overhead [2]. Most of the designed MAC protocols are designed based on schedule-based and contention-based concepts. Schedulebased or TDMA protocols reduce the duty cycle of sensor nodes because the transmission and sleeping periods are defined and scheduled in advance. Moreover, all aforementioned wasted energy sources are avoided or diminished because nodes transmit or receive in their own allocated slots.

Any protocol, whether built on the basis of schedule or contention, should consider the physical layer properties and implementation topology involved. One of the most challenging topologies is the long chain topology since it covers a large scale of region and requires multihops communication. Thus, chain topology is commonly used for monitoring and surveillance applications, where the sensor is deployed linearly. However, a few MAC protocols proposed particularly for this type of topology are contention-based MAC protocols as in [3].
One application for a chain topology is a wireless sensor network where the sensor nodes are embedded in a road and are used to monitor traffic [4]. This type of network can provide a source of data for intelligent transport systems (ITS). This application requires the MAC protocol to consider energy-efficiency as the nodes may be battery powered. In addition, the physical layer properties should be considered including the choice of wireless technology, the radio frequency, the transmitter power and the propagation loss.

The aim of this paper is to introduce a new MAC protocol that built specifically for WSNs deployed in fixed chain topology. This protocol considers gerund level communication pattern for the deployed nodes. To minimise the frame length, it also takes into account the deployment and the physical properties to provide a simultaneous transmission for multi-nodes. The scheduled simultaneous transmission will be relying on SINR of ground level propagation model (GLPM) in chain topology. In this paper, we model a multihop wireless network as a set of stationary nodes.

The proposed protocol will consider a topology that consists of nodes deployed linearly. The deployed nodes will thus communicate with road side units (RSUs) over multihop. However, the spatial reuse technique allows a one-time slot to be used by several transmitters as long as no interference is involved. Therefore, the spatial reuse technique will be utilised to reduce frame size, as well as delay and improve the Packet Received Ratio PRR. In this study, we propose a schedule-based MAC protocol for the chain topology. The novelty of this protocol, therefore, is the introduction of a new signal-to interference-noiseratio (SINR) model that rely on a ground-level propagation model (GLPM). And then the protocol introduces a time slot allocation scheme that guarentees free collision, and it improves the delay in the long chain topology.

The rest of the paper is structured as follows: Section II provides a review of the literature on MAC protocols for wireless systems. Section III describes the system model of the MAC protocol, and Section IV discusses the technical details of the proposed MAC protocol. Section V presents the simulation and evaluation of the proposed protocol. Finally, Section VI summarises the results of the evaluation.

\section{RELATED WORK}

A large number of MAC protocols have been proposed for WSNs [4-7] for different applications. The mechanisms of these protocols can be classified into two major types: 
contention and scheduled based. Since the main concern is the schedule based protocols, this type will be investigated deeply with considering the protocols designed for linear topology.

A few schedule-based protocols have been proposed for chain or linear topology. These protocols used different techniques to generate a feasible transmission schedule with free-interference. As in [6], the proposed protocol designed specifically for chain topology, where the directional antenna was used to enhance the performance. The directional antenna introduced an advantage by reducing the space between any two nodes transmitting simultaneously. This advantage gained since the back lobe of the directional antenna has much smaller range effect of interference than the omnidirectional antenna. The graphbased approach was used to build the schedule of the simultaneous transmission. As well as the performance demonstrated by conducted simulation. However, the configuration of simulation considers ideal channel and path loss attenuation caused by free space path loss model. This assumption does not reflect the practical scenario. As well, the graph-based method has been used to determine the interference range, this method does not estimate the interference range precisely, especially when the directional antenna is used.

In [4], the author proposed a MAC protocol for wireless communication on linear topology. The spatial reuse of time slot was considered as main concept of this protocol. The communication between WiWi nodes is synchronised, based on fixed size packets. The presented concurrent transmission schedule has been built based on graph approach. The main scoop of this protocol was likely to be on the fault tolerant and energy consumption[4]. However a straightforward schedule for concurrent transmission investigated briefly. Therefore, most of the parameters that effect on the concurrent transmission schedule have been skipped such as interference model, propagation model.

In [5], an analytical framework presented to investigate co-channel spatial reuse in dense wireless ad-hoc networks based on RF propagation models for some common network topologies. Chain topology of wireless has been considered in this study, and the minimum distance between two simultaneous transmissions has been calculated. Physical layer properties considered in this calculation to identify $\mathrm{k}$ value by calculate SIR (Signal Interference Ratio). The $\mathrm{k}$ value represents the minimum separation distance between any concurrent transmissions in chain topology with free-interference. However, the separation distance validated but without transmission schedule. As well, the used propagation model in this study is FSPL which does not reflect most the practical scenarios. Moreover the model considers only the interference factor without the noise, it is important to consider SINR to include the noise factor, since it affects $\mathrm{k}$ value. Therefore, this framework can be considered for node scheduling (where all the node broadcasting and share the distention) but not appropriate for link scheduling[7].

As noticed most the reported protocols rely on graphbased approach to build schedule of transmission to enable spatial reuse TDMA and maintain the interference. This approach used to avoid the complexity, but it sacrificing by the accuracy of avoiding the interference. The needed accuracy is likely provided by SINR model that is consider the physical layer properties and Signal Noise Ratio (SNR). Even though, these protocols used SINR model to estimate the interference, it still not accurate enough to build free-interference spatial TDMA protocol for ground level communication. This referred to that; the existing protocols assume a Free Space Path loss model to calculate SINR. However, these proposed protocols [4-8] are not suitable to be implemented in a road-based wireless sensor chain topology, where the used propagation model is designed specifically for ground level communication .Therefore, these reported protocols does not take into account the system topology and properties of the physical layer.

\section{SYSTEM MODEL OF THE MAC PROTOCOL}

A fixed-chain WSNs in a chain topolgy have applications in road-based network monitoring for smart cities and intelligent transportation systems, mining and in bridges.

The use of an appropriate MAC protocol responsible for the allocation of wireless channels among sensor nodes [3] is critical to meet the application requirements of chaintype WSNs. WSN Sensor nodes need to minimise energy consumption. This means the transmitter power should be minimised and so the transmission range is limited. They also have limited processing power and storage capacity. Therefore, these factors should be taken into account in the design of a suitable MAC protocol.

\section{A. Propagation model of fixed chain near-ground-level radios}

For road-based sensor applications, the sensors will be installed on the road and will sense when vehicles move over the nodes. A chain topology allows continuous monitoring over a section of the road.

One of the issues in a multihop chain network is the delay taken to transfer data from the sensor nodes to the access point. In this design, the topology is divided into a subchain containing many Road Studs (RS) and RSU nodes. The topology consists of two types of nodes, RSU and RS. These nodes have different functions. The RSU is responsible for collecting the sensed data by the RS nodes and the channel allocation process. The RS node rules are sensing traffic conditions and measuring the average speed prior to sending the collected data to RSUs.

RSU and RS nodes have some technical differences. RSU nodes have more storage, memory and access to a mains power source. By contrast, RS nodes are battery powered which can be augmented by a small solar cell. These differences and limitations need to be considered during uplink and downlink planning and the topology design.

The maximum distance between road studs is a key factor in the system design. Because increasing the chain length will reduce the number of RSUs and the total system cost. The Friis equation (1) is used to calculate the maximum distance for the downlink from the RSU to the RS nodes as this is a line of sight link [9]:

$$
P_{r}=\frac{P_{t} G_{t} G_{r} \lambda^{2}}{(4 \pi R)^{2}}
$$


The range equation can be derived from the Friis equation for a given receiver sensitivity $p_{\text {sens }}[9]$, as in (2):

$$
d_{\text {max }}=\sqrt{\frac{P_{t} G_{t} G_{r} \lambda^{2}}{(4 \pi)^{2} p_{\text {sens }}}},
$$

where $P_{t}$ is the transmitting power, and $G_{r}$ and $G_{t}$ are the antenna gain for the receiver and transmitter, respectively.

The chain length $d_{\max }$ will be affected mainly by the transmitting power of the RSU and the receiver sensitivity of the receiver. Considering these limitations in the topology design stage is important. We referred to our previous study [10] that investigated the relationship between transmission range and antenna height. Our previous work showed the maximum transmission distance with the lowest level of transmitting power for wireless nodes placed on the ground level. In this topology, the maximum transmission range between RS

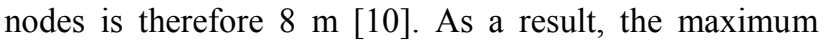
number of nodes in each chain is represented by $\gamma(3)$ :

$$
\gamma=d_{\max } / 8 \text {. }
$$

The uplink can be divided into two categories; the first category has the capability to communicate with the RSU node over one hop on the basis of (2). The second category will rely on a multi-hop path to send its packet to the RSU:

$$
P_{r}=P_{t}\left(\frac{G_{t} G_{r} \lambda^{2}}{(4 \pi d)^{2}}\right) \cdot\left(\frac{h}{d}\right)^{n}
$$

This equation was proposed in a previous work on the propagation model for ground-level wireless communication [10]. It will be used as a basis to define the node spacing and derive the propagation exponent.

\section{B. Interference model}

In order to support simultaneous transmission for several nodes on the same time slot, it is necessary to consider the signal to interference and noise ratio (SINR) interference model which is introduced in [11]. The SINR is a criterion for successful communication (with high probability) if and only if the average SINR at the receiver is above the threshold $\theta$ that assumed $=1$, as in (5):

$$
\operatorname{SINR}=\frac{P_{i} \cdot G_{i i}}{\eta_{i}+\sum_{j=1, j \neq i}^{Q} P_{j} \cdot G_{i j}} \geq \theta,
$$

where $P_{i}$ denotes the transmission power of link $i$ 's transmitter $i_{s} ; n_{i}$ is the receiver noise at link $i$ 's receiver $i_{r}$; $G_{i i}$ and $G i j$ are the link gain from $i_{s}$ to $i r$ and that from link $j$ 's transmitter $j s$ to $i_{r}$, respectively; Q denotes the number of simultaneous transmissions with link $i$; and $\theta$ is the SINR threshold, which is larger than or equal to 1 . Here, the numerator is $G_{i i}$. $P_{i}$ is the received power at $i_{r}$. In the denominator, $G_{i j} p_{j}$ means the attenuated power of $P_{j}$ at $i_{r}$, and it is regarded as the interference power for link $i$; thus, $\sum_{j=1, j \neq i}^{Q} P_{j}$. $G_{i j}$ means the accumulated interferences caused by all other simultaneous transmissions. Because we do not consider fading effects and the possible obstacles in wireless transmissions, the link gain can be represented by an inverse power law model of the link length, i.e. $G_{i i}=1 / \mathrm{d}^{\mathrm{n}}\left(i_{s}, i_{r}\right)$ and $G_{i j}=1 / \mathrm{d}^{\mathrm{n}}\left(\mathrm{j}_{\mathrm{s}}, \mathrm{j}_{\mathrm{r}}\right)$. Here, $d$ is the Euclidean distance function, and $n$ is the path loss exponent that is equal to 12 for an antenna height of $1 \mathrm{~cm}$ on the basis of the Ground Level Propagation Model (GLPM) [10].

Integration of the GLPM with the interference introduces a new interference model that calculates the SINR for ground-level communication. The new SINR contains the blocking factor of ground-level communication to consider the path loss exponent on each antenna height, as in (6):

$$
\operatorname{SINR}=\frac{P_{i \cdot} \cdot\left(\frac{1}{d}\right)^{n}}{\eta_{j}+\sum_{j=1, j \neq i}^{Q} P_{j} \cdot\left(\frac{1}{d}\right)^{n}} \geq \theta,
$$

where the value of $n$ can be calculated by (7) on the basis of antenna height, as stated in [10]:

$$
n=A \cdot h^{3}+B \cdot h^{2}+C \cdot h^{1}+D,
$$

where $h$ is the height of the antenna, and $A, B, C$ and $D$ are the curve fitting constants, which have been studied in [10].

\section{Spatial reuse in a fixed chain topology}

This process involves assigning a one-time slot for multitransmitters to send their packets at the same time. The spatial reuse technique is performed by an accurate schedule of transmission that guarantees free collision.

Node assignment and link assignment are the most common methods to allocate the time slot for multi-users. For multihop communication, link assignment will be used, where the directed link is assigned a slot. A node can thus only use this slot for transmission to a particular neighbour [12].

In the following, we describe the criteria for a set of links to be able to transmit simultaneously with a sufficiently low interference level at the receiving nodes, as shown in Fig. 1. We say that a link $(j, j)$ is adjacent to any other link $(\mathrm{i}, \mathrm{i}) \in L$ if $\{\mathrm{i}, \mathrm{i}\} \cap\{\mathrm{j}, \mathrm{j}\}=\emptyset,(\mathrm{i}, \mathrm{i}) \neq(\mathrm{j}, \mathrm{j})$.

Furthermore, we define $\Psi(L)$ as the union of all adjacent links to the links in $L$. We assume that a node cannot transmit more than one packet in a time slot and that a node cannot receive and transmit simultaneously in a time slot. Alternatively, we say that a set of links $L$ and the set of its adjacent links $\Psi(L)$ must be disjoint:

$$
L \cap \Psi(L)=\emptyset \text {. }
$$

We also require that the SINR value be sufficiently high for reliable communication:

$$
\operatorname{SINR}(i, j) \geq \theta \forall(i, j) \in L \text {. }
$$

If the above two conditions, (8) and (9), hold for a set of links $\mathrm{L} \in \mathrm{L}$, we say that the links in $\mathrm{L}$ can transmit simultaneously.

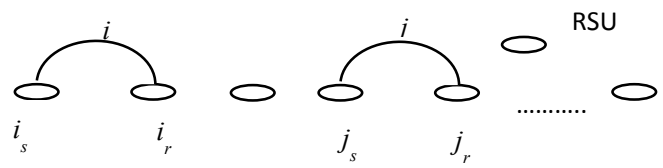

Figure 1: Link assignment for the reused slot

One of the essential tools to build a feasible schedule is the ranking function. This function indicates the preferred order of transmissions, and then produces a feasible schedule [7].

$$
\mathrm{R}_{i}^{H}=R_{i}(\bmod H)
$$


where $H$ is a constant usually larger than or equal to three, where this values reflect the minimum number of hops between any concurrent transmission that defined by interference model [7, 13].

The rank function is used to calculate the transmission order in every slot. Suppose this is used in slot 1 , every node will be represented by $\mathrm{R}_{i}^{H}$, as in (10). In calculating the node transmission order, the first node that has a packet to send will be considered in rank value $\mathrm{Ri}=1$. Once the node has finished its transmission in slot 1 , in the next hop, node 1 will be terminated from the rank assessment, so node 2 will obtain rank number 1 . This process will be repeated in every slot as in algorithm 1 .

$$
\mathrm{R}_{i}^{H}=\left\{\begin{array}{lr}
1, & \text { slot of transmission } \\
2, & \text { slot for receiving } \\
0, & \text { slot for sleeping }
\end{array}\right.
$$

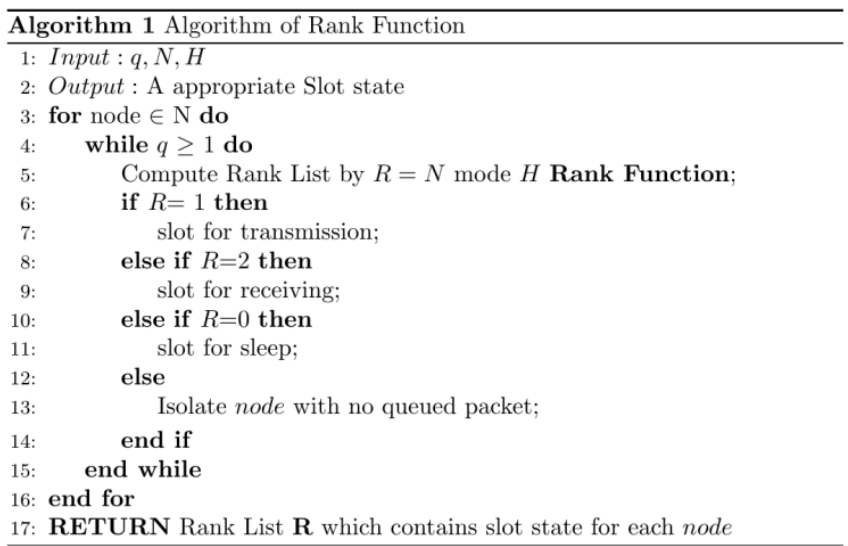

The new ranking function $\mathrm{R}_{i}{ }^{H}$ is performed in every slot. Suppose link $j$ follows link $i$ on the path, such that $R_{i}<R_{j}$. Then, in the proposed transmission ordering, $i$ will transmit before $j$. The identified bound of the SINR in (6) and the transmission order by the rank function in (10) are important keys to scheduling the links with free collision. By implementing these aforementioned equations in a small chain topology, then we can derive an equation that determines the minimum number of required slots, as in (12).

$$
S_{f}=(n * H)-H
$$

Where $\mathrm{n}$ is a number of nodes in the chain, and $\mathrm{H}$ is the constant value representing the number of hops between two transmitters that are using the same slot.

When the chain topology contains some nodes that have the capability to reach the RSU node over one hop, the minimum number of required slots is calculated by (12):

$$
S_{\text {total }}=\left(n_{f}^{*} H\right)+n_{c}
$$

Where $n_{f}$ is the number of nodes using multihop to reach the RSU, and $n_{c}$ represents the number of nodes that have the capability to arrive at the RSU by one hop.

Another important parameter that should be calculated is the slot length. The slot length is the time allocated to transmit a certain amount of data or packets. The appropriate slot length can be determined on the basis of the number of nodes calculated by (3) and the defined frame length, as in (13).

$$
S_{l}=f / S_{\text {total }} \text { sec, }
$$

Where $f$ is the frame length, $S_{l}$ is the suitable slot length and $S_{\text {total }}$ is the minimum number of required slots defined by (12). Once the transmission order, a number of slots and slot length are defined, we will move to defining the assumptions. One of these assumptions is data rate. The assumed data rate $(\alpha)$ is $250 \mathrm{kbps}$; this rate is selected because it is sufficient to transfer the data generated in the road-based wireless sensor network scenario. The data rate and slot length are required to calculate the packet size. As a result, the maximum packet size is:

$$
P_{\text {siz }}=S_{l} * \alpha \text { kbit. }
$$

Therefore, we can calculate the delay in the chain topology, as in (15):

$$
D=\left(\frac{S_{f}}{c_{h}}\right) * S_{l}
$$

where $S_{f}$ is the number of required time slots to deliver a packet from the transmitter to the sink, and $S_{l}$ is the slot length of each slot ms. $C_{h}$ represents the number of chains following the sink.

\section{IV.}

\section{PROPOSED MAC PROTOCOL}

Time division multiple access is a contention-free medium-access protocol. The channel bandwidth shared by all nodes in the network is partitioned into time slots for dedicated use among such nodes. Each node transmits data only during this dedicated time slot allocated to it. In the proposed MAC protocol, the clocks of the nodes are assumed to be perfectly synchronised.

\section{A. Time slot allocation scheme}

The allocation scheme describes the technique used to allocate a time slot for each of the nodes. This scheme is the key to building the schedule of allocated slots. It mainly relies on the rank function in (10) to assign a onetime slot for several nodes. At the same time, it considers the SINR, to calculate the minimum separation between any concurrent transmissions to avoid any interference. The number of nodes and the frame length should be considered to determine the time slot allocation. These variables comprise the input allocation process to calculate the slot length by (13). After the slot length is determined, each time slot will be assigned to several nodes on the basis of the rank function, which considers the value of $H$ to be larger than or equal to three bases on the interference model. The output of the allocation scheme is shown in Fig. 2; the time slot is allocated to several nodes to allow simultaneous transmission. The schedule identifies the node state in each slot.

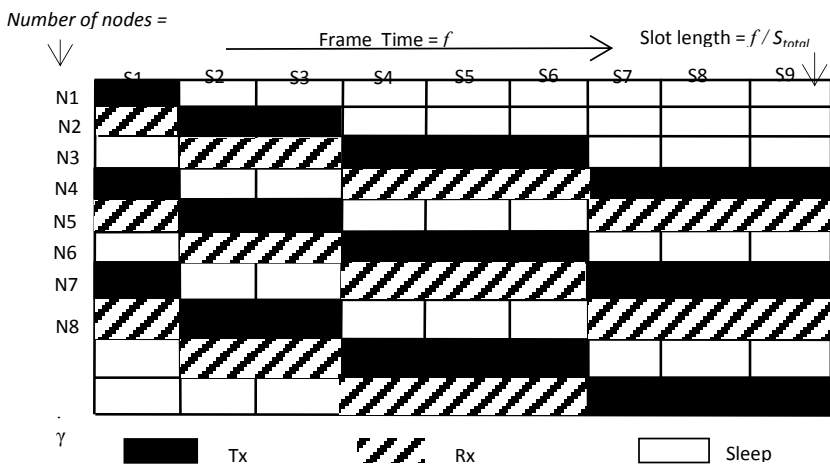

Figure 2: Time slot allocation scheme 
The first slot is assigned to nodes number one, four, seven and ten, to use for transmission Tx. The same slot will be allocated to other nodes to receive the transmitted packet by their neighbours. The rest of the time slots will be allocated to a sleep mode to minimise the energy consumption. This scheme will guarantee efficiency in slot usage and reduce the frame length.

\section{B. Protocol specification and algorithm}

The proposed protocols rely on the network topology and information on the physical layer. The protocol operation is divided into two phases.

Phase one: The protocol requires the following physical information: the number of nodes, the node spacing and the antenna height. This information is needed to calculate the value of the SINR on the basis of GLPM. Therefore, the new SINR metric will consider the path loss exponent of the ground-level propagation level. Then, to avoid any interference, it is essential to ensure that the SINR value is larger than the SINR threshold. Doing so will guarantee that the required criteria of simultaneous transmission in one slot are met as discribed in the proposed algorithem in Fig.4.

Phase two: Once the SINR value and $H$ value are defined, the protocol starts to request some initial information, such as the frame length $f$ and number of nodes. Then, the appropriate slot length will be calculated with the use of this information, as in (13). In each slot, the rank function will then be used to derive the transmission order for each node. The outcome of this function will indicate the node state in each slot, as shown in Fig.2. The final step in this phase is releasing the transmission schedule to all the nodes in the topology. As a result, the simultaneous transmission will be identified without any collision.

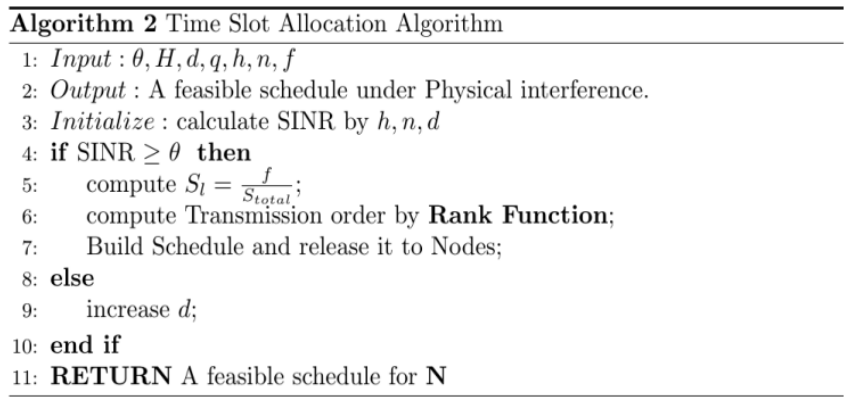

The algorithm 2 describes the operational concept of the proposed protocol. It includes the specification of phases one and two.

\section{MAC protocol design}

The protocol determines the operation of the MAC protocol in each node and how it manages the time slot assignment. The MAC protocol consists of three main parts, which work integrally to deal with the scheduling of transmission without any conflict.

Central MAC: The entire data packets ready for transmission by the node are sent down either from the IP to the Central MAC. Upon receiving the packets, the Central MAC queues them and waits for the turn of these nodes to transmit. As soon as these nodes have their turn to transmit, the Central MAC looks up the MAC queue for any queued packets. It then iteratively de-queues packets, attaches the MAC header and trailers and then sends them to the simple-wireless-channel. Before sending them, the Central MAC calculates the transmission time required on the basis of packet size and data rate. It adds up the transmission times of all the packets sent and compares these with the slot time provided by the TDMA Controller. If the Central MAC could not transmit any more packets in a particular slot, the loop terminates, and further transmissions stop. The simple-wireless-channel forwards the packets to the node address defined in the header.

MAC controller: The TDMA controller handles all scheduling aspects of the protocol. It initiates spatial time division multiple access (STDMA) sessions and authorises the nodes to transmit in the slots it specifies. The number of slots allocated for transmission, along with the slot durations, is provided to it by the allocation scheme and rank function. Then, the MAC Controller maintains a list of MAC pointers associated with all the nodes. Based on the slot assignment provided by allocation scheme, this list is populated by the TdmaHelper class before the simulation starts. After the simulation starts, the MAC Controller initiates the scheduling of STDMA sessions on the basis of node IDs. It calls the Central MAC from its list of MAC pointers and instructs the nodes that they can transmit for a particular slot time on the basis of the rank function. As soon as the transmission slot for such nodes is complete, the MAC Controller waits for GuardTime, and then the MAC controller calls the nodes again to perform the rank function to determine the transmission order. Once all the nodes from the list are assigned a transmission slot, the controller waits for the InterFrameTime before starting with the same procedure again as in Fig. 3.

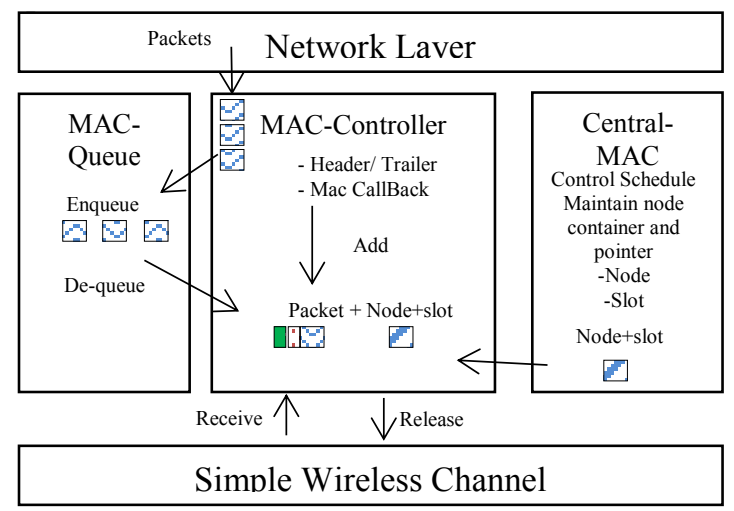

Figure 3: Protocol component

MAC queue: The TDMA maintains a drop-tail queue to store the packets received from the network layer until it gets its transmission slot. The attributes that can be modified for this class are the MAC queue length and MAC queue time. Thus, all the packets trying to be enqueued after the queue size reaches the MAC queue length are dropped; the packets stored in the queue for a time interval longer than the MAC queue time are also dropped. 


\section{SimUlation AND PERFORMANCE ANALYSIS}

In this section, we show the simulation results of our proposed TDMA protocol implemented on chain topology with the use of the NS-3 network simulator.

Table 1: Simulation variable values

\begin{tabular}{|l|c|}
\hline Parameters & Value \\
\hline Slot length & $10 \mathrm{~ms}$ \\
\hline Frame length & $1 \mathrm{~s}$ \\
\hline Number of nodes & 75 \\
\hline Number of Slot & 100 slot as in (12) \\
\hline Packet size & 312 bytes \\
\hline Data rate & $250 \mathrm{kbps}$ \\
\hline Ptx for RSUs & $100 \mathrm{~mW}$ \\
\hline Ptx for RSs & $2 \mathrm{~mW}$ \\
\hline
\end{tabular}

The parameter values in Table 1 are evaluated with the use of the simulator to validate the proposed protocol. In this scenario, one chain is considered; this chain is controlled by one RSU node, and its transmitting power is $100 \mathrm{~mW}$, so we use (3) to calculate the number of nodes. The node spacing is $8 \mathrm{~m}$ based on the GLPM used in (4) [10]. Thus, the number of nodes is 75 nodes, and the frame length is assumed to be $1 \mathrm{~s}$.

Based on (1), (2) and the node capability, the topology will be divided, as shown in Fig. 4.

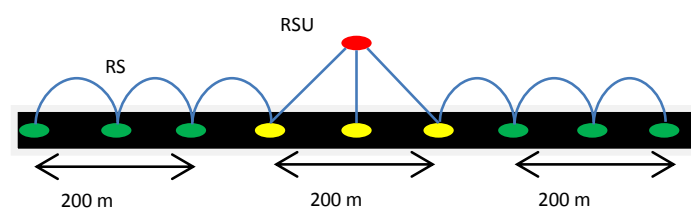

Figure 4: Simulation network

In the downlink, the RSU node can reach all the RS nodes in the chain because all RS nodes are located in its coverage range. Regarding the uplink, the nearest 25 nodes to the RSU can communicate with the RSU over one hop on the basis of (2) and the technical capability in Table (1). The rest of the RS node will reach the RSU node over multi-hops.

Equation (11) is used to calculate the slot length for each node, which is $1 / 100 \mathrm{~s}$, and (12) determines the maximum packet size for each transmission for each node, which is 312 bytes. Based on the packet size, the magnetic sensor can collect 100 samples/car, and the sample size is 8 bit $=800 \mathrm{bits} / \mathrm{car}$; if the packet size is 2,500 bits or 312 bytes, then each packet can carry the information of 3 sensed cars because $2500 / 800=3.12$ cars $/ \mathrm{sec}$. The simulation ran several times and considered two MAC techniques: classic TDMA and the proposed spatial TDMA.

\section{A. Proposed Spatial TDMA vs. Classic approach TDMA}

The efficiency of a protocol depends on several factors, including delay and the dropped packets ratio. Here, two types of protocols are assessed: classic TDMA and developed Spatial TDMA are implemented in the chain topology. We used the same input value for both to measure the delay and the dropped packet ratio.

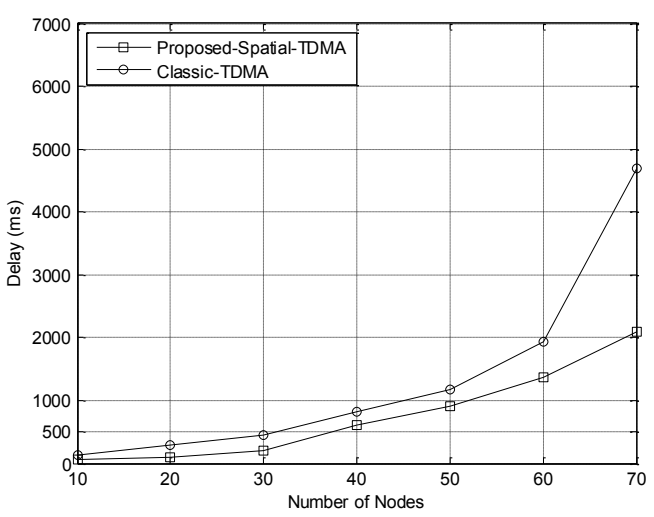

Figure 5: Compared Delay of Classic TDMA and Proposed Spatial TDMA in the chain topology

As shown in the above Fig. 5, the delay has been measured in a chain topology based on (15). It compares the delay between scheduled concurrent transmission TDMA and classic TDMA:

The experiment was conducted on a different node number and with one packet transmitted. The result in Fig. 5 shows that in the chain topology, the delay of the classic TDMA increases rapidly when the number of nodes increases. By contrast, delay of the proposed spatial TDMA still in an acceptable level. This phenomenon reffered to that the classic TDMA is desinged to assign one time slot for each node, so when it be used on chain topology where the nodes required to transmit its packets and the forwarded packets, the number of allocated slot for each frame will increase cumulatively as in (16).

$$
N_{i}=\frac{n(n-1)}{2}
$$

Where $N_{i}$ is the number of required slots, and $n$ is the number of nodes [14]. This increase of allocated slots will lead to increase the frame length which is significantly cause the delay. Therefore, proposed protocol reduces the end-to-end delay by take the advantage of spatial technique to reuse time slots for simultaneous transmission for nodes that are outside the radio interference range, and reduce the delay.

Fig. 6 clearly shows a ratio of dropped packets in classic TDMA larger than Proposed Spatial TDMA. To simplify the scenario, a different number of nodes are considered, and in each node, one packet is transmitted via fixed data rate. Typically, the number of scheduled packets proportion with the number of node in the chain, so increase number of nodes lead to increase number of scheduled packets. In Classic TDMA, this requires at least one slot is assigned for each node.

The rapid increase of dropped packets in classic TDMA is referred to that increases the number of nodes leads to increase the number of assigned slots. Therefore, the frame length will be expanded, and then some the schedule node will excess the accepted level of delay that is predefined on the queuing delay specification. 


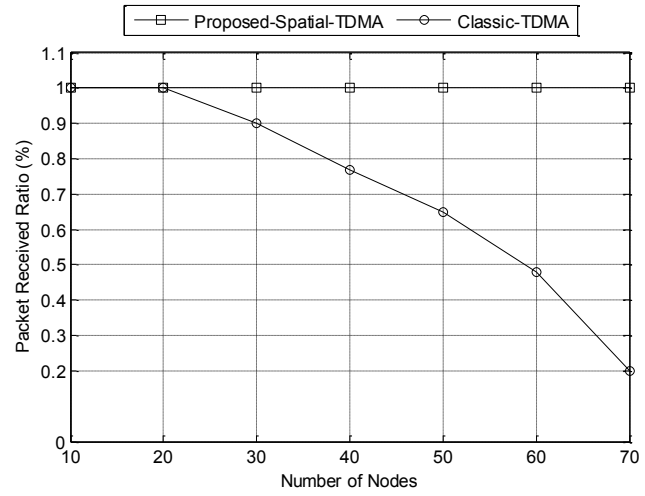

Figure 6: Compared packet dropped ratio of Classic TDMA and Proposed Spatial TDMA in the chain topology

As result, some scheduled packet will be dropped due to it exceeding the acceptable queuing delay. By contrast, the proposed spatial TDMA delivered most of the sent packets despite a large number of transmitters. This success in the delivery of packets is due to the used allocation process following spatial reuse technique that allow multi users transmit its packets simultaneously, and then this technique minimise the frame length. As well as the proposed protocol consider the neuter of topology where the multihops communication is required, and the physical layer properties include the SINR based on ground level propagation level [10], where the interference is avoided. All these significant factors have been considered on the proposed spatial TDMA protocol, and this improvement reflected on the PRR and delay.

\section{B. Proposed TDMA vs. WiWi and Dis-MAC}

In this section, a numerical analysis and simulation are conducted to predict the delay of the proposed protocol and compare it with those of the other spatial TDMA protocols in Fig. 7.

Spatial reuse technique has been considered in several TDMA protocols in order to reduce the end-to-end delay. However, the spatial reuse concept has been implemented and redesigned for these protocols base on different approach such as graph-model and SINR model [4-6]. These protocols applied on chain topology where the ground level communication considered.

The results in Fig.7 reflect that, a better delay can be achieved with the proposed spatial TDMA protocol compared with transmission schedule for spatial TDMA protocols in $[4,7]$.

This difference in Fig. 8 is referred to that these transmission schedules has been designed base on graphbased where the physical layer properties are not considered and that effect the accuracy of estimated spacing between any concurrent transmission. As well as the proposed transmission schedule that has been built base on SINR approach or physical model still comes with higher delay, because the considered propagation model is FSPL where this model requires larger spacing between any simultaneous transmissions. At the end, the effect of using FSPL is reflected on the transmission schedule.

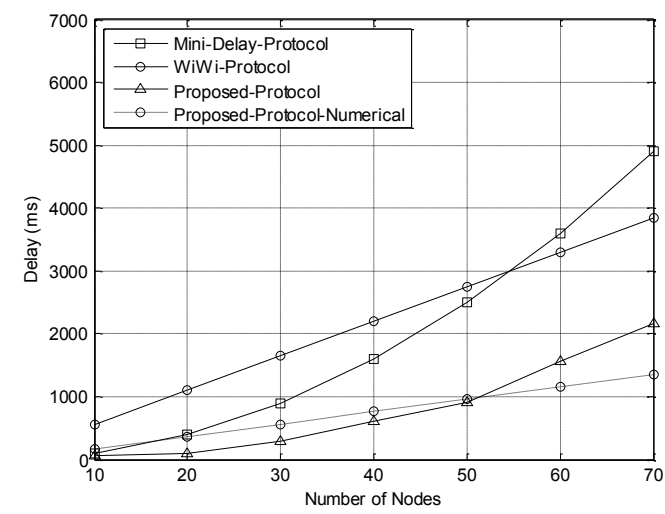

Figure 7: End-to-end delay of a different TDMA approach implemented in the chain topology

Thus, the proposed spatial TDMA achieved lower level of delay, due to it has been built base on SINR-based that more accurate than graph-based, because it consider physical layer properties to estimate the interference range. In addition, the proposed spatial TDMA protocol relies on GLPM instead of using FSPL. Using GLPM to calculate SINR is essential to define the physical boundaries and the interference range precisely for ground level communication. As a result, the interference can be avoided with shorter separation distance between simultaneous transmissions. And, the combination of GLPM and SINR will result in produce much smaller frame length that reduces the delay.

\section{CONCLUSION}

In this paper, we have proposed our MAC protocol for ground level wireless sensors in a fixed chain topology. The SINR model is combined with the GLPM to build an efficient concurrent transmission schedule. This combination takes into account the physical properties and achieves the highest level of slot reuse. This efficiency is achieved with the use of the rank function to determine the transmission order, which serves as the key to building a feasible transmission schedule. Numerical analysis has been conducted to optimise the topology, which includes the chain length and the simultaneous transmission range. Additionally, we have proposed equations to calculate the minimum number of required slots for each chain. To evaluate the proposed protocol, a numerical analysis has been carried out, and the performance of the protocol is compared with those of the other TDMA protocols. Moreover, a simulation scenario has been designed to assess the performance of the proposed protocol compared with the classic TDMA. The results show the ratio of dropped packets in the proposed TDMA is stable compared with classic TDMA where the ratio of dropped packet increases dramatically. In addition, the proposed delay metric shows that the proposed protocol has a lower delay than the classic TDMA and STDM.

\section{REFERENCES}

[1] H. Gong, M. Liu, G. Chen, and X. Zhang, "A study on event-driven TDMA protocol for 
wireless sensor networks," EURASIP Journal on Wireless Communications and Networking, vol. 2010, p. 9, 2010.

[2] S. Rahman, M. Ahmad, and S. A. Bazaz, "A new energy-efficient TDMA-based MAC protocol for periodic sensing applications in wireless sensor networks," International Journal of Computer Science Issues, vol. 9, pp. 214-221, 2012.

[3] C. Fang, H. Liu, and L. Qian, "LC-MAC: An Efficient MAC Protocol for the Long-Chain Wireless Sensor Networks," in Communications and Mobile Computing (CMC), 2011 Third International Conference on, 2011, pp. 495-500.

[4] D. De Caneva and P. L. Montessoro, "A synchronous and deterministic MAC protocol for wireless communications on linear topologies," Int'l J. of Communications, Network and System Sciences, vol. 3, p. 925, 2010.

[5] X. Guo, S. Roy, and W. S. Conner, "Spatial reuse in wireless ad-hoc networks," in Vehicular Technology Conference, 2003. VTC 2003-Fall. 2003 IEEE 58th, 2003, pp. 1437-1442.

[6] T. Karveli, K. Voulgaris, M. Ghavami, and A. H. Aghvami, "DiS-MAC: A MAC protocol for sensor networks used for roadside and highway monitoring," in 2009 International Conference on Ultra Modern Telecommunications \& Workshops, 2009, pp. 1-6.

[7] P. Djukic and S. Valaee, "Link scheduling for minimum delay in spatial re-use TDMA," in INFOCOM 2007. 26th IEEE International Conference on Computer Communications. IEEE, 2007, pp. 28-36.

[8] I. F. Akyildiz, W. Su, Y. Sankarasubramaniam, and E. Cayirci, "A survey on sensor networks," Communications magazine, IEEE, vol. 40, pp. 102-114, 2002.

[9] B. A. Black, Introduction to Wireless Systems: Prentice-Hall, 2008.

[10] J. Alshudukhi, S. Ou, and P. Ball, "A ground level radio propagation model for road-based wireless sensor networks," in Communication Systems, Networks \& Digital Signal Processing (CSNDSP), 2014 9th International Symposium on, 2014, pp. 146-151.

[11] P. Gupta and P. R. Kumar, "The capacity of wireless networks," Information Theory, IEEE Transactions on, vol. 46, pp. 388-404, 2000.

[12] J. Grönkvist, "Interference-based scheduling in spatial reuse TDMA," 2005.

[13] P. Djukic and S. Valaee, "Delay aware link scheduling for multi-hop TDMA wireless networks," IEEE/ACM Transactions on Networking (TON), vol. 17, pp. 870-883, 2009.

[14] S. Razak, V. Kolar, N. B. Abu-Ghazaleh, and K. A. Harras, "How do wireless chains behave?: the impact of mac interactions," in Proceedings of the 12th ACM international conference on Modeling, analysis and simulation of wireless and mobile systems, 2009, pp. 212-220. 